\title{
LA ÉTICA DISCURSIVA Y EL PROBLEMA DEL CONOCIMIENTO PRE-LINGÜÍSTICO*
}

\section{Andrés Crelier **}

Resumen: El trabajo problematiza la relación entre el discurso, la base de fundamentación de la ética discursiva, y las capacidades cognitivas pre-lingüísticas. En primer lugar, se critican diversas formas de idealismo lingüístico que suponen que toda capacidad cognitiva depende del lenguaje y se defiende un idealismo "débil", según el cual el conocimiento no lingüístico es virtualmente expresable mediante el lenguaje. En segundo término, se sostiene que entender de este modo lo pre-lingüístico permite aplicar el principio de la ética discursiva a agentes incapaces de usar el discurso, como los niños pre-lingüísticos o determinados animales cercanos evolutivamente al hombre.

Palabras clave: ética discursiva, conocimiento, idealismo lingüístico

Abstract: This paper analyses the relation between discourse, which is the justification basis of Discourse Ethics, and the pre-linguistic cognitive capacities. First, we criticize different forms of linguistic idealism and defend a weak version of idealism according to which non-linguistic knowledge is virtually expressible by means of language. Secondly, we claim that in this way the principle of the Discourse Ethics can be applied even to agents incapable of discourse, such as prelinguistic children and certain animals closely related to human beings.

Key Words: Discourse Ethics, Knowledge, Linguistic Idealism

\section{Introducción: sobre el carácter "expansivo" del principio ético- discursivo}

Para la ética discursiva, todo aquel que entra en el discurso, entendido como intercambio argumentativo con fines teóricos o prácticos, comparte

\footnotetext{
* Una primera versión de este trabajo fue leída en el VI Coloquio Latinoamericano sobre Ética del Discurso, organizado por la Fundación ICALA en noviembre de 2011 en Río Cuarto. Los comentarios y la discusión me han permitido reelaborar mis ideas y darles la forma del presente artículo.

** Doctor en Filosofía por la Universidad Nacional de La Plata. Es investigador asistente del CONICET y Profesor Adjunto de las cátedras de Filosofía Contemporánea y Filosofía del Lenguaje en la Universidad Nacional de Mar del Plata. Dirección electrónica: andrescrelier@yahoo.com.ar
} 
necesariamente una serie de presupuestos, algunos de los cuales poseen una naturaleza moral. Dicho de manera aproximada, se trata de la exigencia de tener en cuenta como personas con iguales derechos no sólo a quienes intervienen activamente en el discurso sino a todos los afectados por las decisiones que se tomen en caso de conflicto práctico.

Como es sabido, existen diversas cuestiones problemáticas referidas a este principio y a la ética discursiva, tanto en el campo de la fundamentación ética como en el de la aplicación. En este trabajo me centraré en una de ellas, asumiendo sin embargo de entrada la validez de esta propuesta éticodiscursiva y de su principio moral.

Para reconstruir el problema al que me abocaré, empezaré señalando que este principio tiene necesariamente un carácter "expansivo" en al menos dos sentidos, uno "interno" y otro "externo" al lenguaje. El primero es interno al lenguaje en general, pues se trata de afirmar su validez también en el marco de los usos no argumentativos -dicho técnicamente, no "discursivos"- del lenguaje. Así, el principio tiene validez para quien cuenta un relato, expone una descripción o da una orden. La razón de esto es a su vez doble: por un lado, se trata de concebir a la argumentación como implicada en todo uso de lenguaje en el que se pueda dar y ofrecer justificaciones, las cuales se refieren ante todo a puntos de vista diferentes sobre los actos lingüísticos realizados. Así, podemos dar o pedir razones acerca de diversos elementos de un relato, los fundamentos de una orden o la adecuación de una descripción. Por el otro, se trata del supuesto de que un agente que dispone en general de la capacidad de hablar un lenguaje es también, de manera necesaria, un agente capaz de argumentar, de entrar en el mencionado intercambio en su actualización más cabal.

El sentido "externo" del carácter expansivo de la validez del principio ético-discursivo atañe ya no al lenguaje en general sino al ámbito de la acción extra-lingüística en general. En efecto, para que resulte moralmente relevante no sólo se debe pretender que el principio resulta válido para todo agente lingüístico capaz de argumentar y tomar decisiones mediante el lenguaje sino también para el que actúa en general. Es decir, no sólo para quien realiza una "reflexión estricta" (Kuhlmann), "aquí y ahora” (Böhler), interrumpiendo acaso su acción en el "mundo de la vida" y entrando en una argumentación para decidir con mejores razones cómo actuar en caso de 
conflicto práctico, sino para el que actúa sin argumentar $y$, al menos en apariencia, sin hacer uso del lenguaje.

Así, si bien la fundamentación del principio es argumentativa y en tal medida lingüística, su ámbito de aplicación abarca a todos los seres morales con capacidad lingüística, aun en los momentos en que esta capacidad no se encuentre realizada de manera actual. De hecho, las faltas morales graves son cometidas usualmente por agentes que no están argumentando al momento de actuar, lo cual no debe hacer mella, naturalmente, a la validez del principio moral. Estos agentes están sometidos al principio éticodiscursivo aun cuando no estén ejercitando su capacidad lingüística y específicamente argumentativa.

En este sentido, el carácter expansivo de la validez del principio éticodiscursivo se ha plasmado en la valiosa noción de un "discurso virtual acompañante" de las acciones humanas con sentido. ${ }^{1}$ Los actos realizados en el mundo de la vida por parte de seres con capacidad lingüística y argumentativa son virtualmente justificables desde un punto de vista moral. El principio moral del discurso abarca no sólo a quien realiza o impugna la fundamentación y a quien se mueve de manera actual en el medio del lenguaje, sino a los seres humanos que actúan en general, incluyendo a los niños incapaces todavía de hablar y a aquellos que han perdido esta capacidad. En nociones de esta clase se basa no sólo una perspectiva ética como la que aquí se discute sino gran parte del derecho, donde la justificación lingüística cumple un rol relevante incluso cuando se aplica a acciones realizadas en un marco no lingüístico (aunque la atribución de responsabilidad moral es en el derecho una cuestión empírica, que se resuelve por ejemplo consultando a un psiquiatra acerca del estado mental del acusado, o a un perito acerca de la cantidad de alcohol en sangre, etc. En filosofía, por el contrario, se abre un terreno de discusión conceptual acerca de los límites del ámbito de validez).

Llegados a este punto se ha producido una distancia, todavía sutil, entre uso actual del lenguaje (y de la argumentación, pues supongo que estar

${ }^{1}$ Cf. Böhler, Dietrich, Rekonstruktive Pragmatik, Frankfurt am Main, Suhrkamp, 1985, y Damiani, Alberto, Handlungswissen. Eine transzendentale Erkundung nach der sprachpragmatischen Wende, Freiburg / München, Karl Alber, 2009. 
en el lenguaje es estar en el espacio en el que se pueden dar razones) y la capacidad lingüística no actual, es decir, no actualizada. Y surge un problema que puede formularse como sigue: dado que es preciso atribuir responsabilidad moral no sólo a quienes están argumentando de una manera actual, se necesitan criterios claros para determinar qué agentes consideramos virtualmente capaces de este uso del lenguaje, donde el principio moral se encuentra claramente presupuesto.

Argumentantes virtuales son los niños, pero ¿desde qué edad? Argumentantes que han perdido esa capacidad son quienes padecen ciertas enfermedades o la han perdido por causas naturales de vejez, pero ¿ya por eso los dejamos de considerar agentes morales? Aquí aludimos todavía a capacidades próximas al uso actual del lenguaje, pero ¿qué sucede con aquellos animales cercanos al hombre que comparten con éste procesos cognitivos complejos? ¿Debemos excluirlos de la moralidad en todo sentido? $Y$ si, mediante un ejercicio fundamentado de la imaginación, representamos sus deseos, intereses y acciones comunicativas de manera lingüística, ¿ya por eso los debemos incluir en la esfera moral?

Existen diversos modos de abordar estas preguntas, las cuales atañen a los alcances de la validez del principio ético discursivo respecto de los agentes virtualmente $-\mathrm{y}$ no actualmente- discursivos. Mi intención, para explorar la relación entre lo actual y lo virtual, es partir de la siguiente estipulación: dado que lo virtualmente argumentativo no se equipara, ex bipothesi, a un uso actual del lenguaje, lo consideraré "pre-lingüístico". El sentido preciso de esta denominación quedará claro -al menos así lo espero- a lo largo de este trabajo.

Como las preguntas anteriores ponían en evidencia, no todos los seres morales son seres lingüísticos en un sentido pleno. De este modo, para la ética discursiva la capacidad lingüística efectivamente ejercida es una condición suficiente para determinar que estamos ante un agente moral, pero no es una condición necesaria, pues puede en principio haber seres morales no lingüísticos o que no actualicen esa capacidad. Dentro del universo posible de seres morales no lingüísticos excluiré, en la presente discusión, aquellos cuya conformación biológica no incluye ninguna de las capacidades relacionadas con el lenguaje. Sí interesan, por el contrario, aquellos agentes que poseen capacidades "racionales" o cognitivas complejas, pues podemos suponer que estas capacidades son virtualmente 
lingüísticas dado que en los seres plenamente lingüísticos es el lenguaje el que las vehiculiza.

Exploraré precisamente la relación entre estas capacidades, entendidas como pre-lingüísticas, y el lenguaje. En el caso de los humanos, se trata por ejemplo de poder identificar y reidentificar objetos, personas y situaciones; de actuar en el sentido de la capacidad de deliberar acerca de medios adecuados para lograr ciertos fines; de realizar inferencias que involucren cosas, personas y situaciones; de tener creencias y actitudes intencionales, de comprender creencias y actitudes intencionales de otros, de comunicarse en base a esta comprensión, etc. Ciertamente, algunas o todas estas capacidades han sido ligadas tan fuertemente con el lenguaje que se ha llegado a sostener que no existen propiamente fuera de esta unión. Sin embargo, la evidencia empírica parece mostrar lo contrario, tanto en seres humanos como en animales. Los niños pre-lingüísticos, por ejemplo, reconocen personas, comprenden el sentido de juegos complejos, extraen conclusiones a partir de situaciones determinadas, interactúan, se comunican, etc. Y claramente los consideramos seres morales antes de que hayan adquirido plena capacidad lingüística.

Mi propuesta es que si podemos establecer un lazo firme entre estas capacidades y la argumentación, esto reforzará la tesis de que la ética discursiva no sólo se aplica a quien está inserto actualmente en un juego de lenguaje argumentativo. Tendremos mejores argumentos para extender el ámbito de validez del principio del discurso más allá de la argumentación actual, hacia aquellos agentes pre-lingüísticos que podríamos considerar cercanos al lenguaje.

\section{Crítica del idealismo lingüístico}

¿Puede realmente afirmarse que las capacidades mencionadas más arriba están presentes incluso en ausencia de capacidades lingüísticas? Ciertamente, la mera enumeración de ejemplos incurre en una petitio si no se ofrece también una justificación filosófica. Pero tampoco basta con afirmar que ejemplos suelen estar cargados de teoría, y rechazarlos por esta sola razón. Hace falta en suma acercarse a la experiencia con alguna interpretación teórica defendible, ya sea específica o general. En este apartado criticaré una posición idealista que no me parece justamente un 
modo adecuado de entender las capacidades en apariencia no dependen del lenguaje.

Empezaré presentando la descripción de algunos fenómenos que sugieren la presencia, en animales no humanos, de una capacidad tan compleja como la deliberación práctica. Concretamente, el científico cognitivo y filósofo Michael Tomasello ha argumentado a favor de la presencia de intencionalidad en simios cercanos evolutivamente al hombre. ${ }^{2}$ Para este investigador, quienes estudian sólo humanos no aprecian la flexibilidad que tienen los simios respecto de la comunicación gestual. En general, la comunicación animal se encuentra fijada y determinada rígidamente por disposiciones genéticas, pero los grandes simios ofrecen una novedad evolutiva en el hecho de mostrar conductas comunicativas mucho más flexibles. El uso de diferentes signos en diferentes ocasiones, dependiendo del estado de atención del receptor (como sucede en la comunicación gestual de los simios), requiere que quien se comunica posea un modelo cognitivo flexible y complejo. Investigaciones recientes prueban, según Tomasello, que los grandes simios y los niños pre-lingüísticos poseen un modelo con esta flexibilidad, lo cual les permite entender a los otros como agentes intencionales, ante todo como agentes que tienen fines y los persiguen hasta alcanzarlos. La evidencia experimental que apoya esto incluye la siguiente:

Cuando un humano le acerca alimento a un chimpancé y luego deja de hacerlo, la conducta de este último es diferente si el humano ha dejado de hacerlo sin razón aparente (en cuyo caso el animal muestra frustración), o si hay algo que se lo impide (en cuyo caso muestra paciencia).

- $\quad$ Cuando un humano o un congénere quiere alcanzar un objeto ubicado fuera de su radio de alcance, el chimpancé lo ayuda, lo cual implica -según Tomasello- comprender el fin perseguido por otro agente.

- El chimpancé criado entre humanos conoce la diferencia entre acciones producidas libremente y acciones forzadas por las circunstancias, pues en contextos adecuados y controlados de experimentación imita tan sólo las primeras.

2 Tomasello, Michael, Origins of Human Communication, Massachusetts, The MIT Press, 2008, p. 43. 
Además de comprender a los demás como agentes que persiguen fines, los niños pre-lingüísticos y los grandes simios comprenden que los otros perciben de modo diferente a ellos mismos (ante todo, que tienen un campo perceptivo diferente). Ambas cosas los habilitan para una forma básica de razonamiento práctico consistente en comprender y predecir lo que hacen y harán los otros. Así, los participantes de experiencias controladas entienden que si sus competidores pueden ver la comida la intentarán alcanzar, y que si no la pueden ver, por ejemplo porque existe una barrera que lo impide, no lo intentarán.

Tomasello concluye: "Esta clase de razonamiento práctico acerca de los otros -en términos de los predicados psicológicos querer, ver y hacer- es la base para todos los tipos de interacción social humana y en primates...".3 MacIntyre arriba a una conclusión similar, afirmando que muchos animales no humanos

se orientan ya por un tipo de razonamiento práctico, el que se manifiesta en el hecho de que asuman que esto es una razón para hacer aquello, un tipo de razonamiento que deber ser caracterizado por analogía con el entendimiento humano. Los delfines, los gorilas y los miembros de algunas otras especies no son puramente sensibles y receptivos para los estímulos que reciben sus sentidos, como tampoco lo son los seres humanos (...) Son capaces de crear relaciones no sólo con miembros de su especie sino también con seres humanos y descifrar las interacciones y propósitos de esos seres humanos, a la vez que expresan sus propias intenciones y propósitos... ${ }^{4}$

Tenemos pues, según estas descripciones: presencia de actitudes intencionales, comprensión de la intencionalidad ajena, deliberación práctica, inferencia, identificación de agentes de la acción, creencias acerca de la intencionalidad y la percepción propia y ajena, entre otras capacidades. Lo relevante es que se trata de agentes que claramente no son capaces de hablar un lenguaje convencional e histórico, recibido de una comunidad real
${ }^{3}$ Ibid., p. 49.
${ }^{4}$ MacIntyre, Alasdair, Animales racionales y dependientes, Barcelona, Paidós, 2001, p. 79. 
de comunicación, compuesto por unidades de sentido recombinables indefinidamente para crear nuevas unidades de sentido.

Los fenómenos descritos parecen hablar por sí mismos. Sin embargo, en parte porque se hallan en un marco de interpretación teórica relativamente sofisticado, en parte porque la interpretación ofrecida es en apariencia antropomórfica (se habla de la "frustración" de los chimpancés), no acallan por sí mismos las objeciones propias de una tradición filosófica marcada por el giro lingüístico y, en general, la tesis de la excepcionalidad humana.

Pero no hay que tomar la mera enunciación de estas objeciones como válida de por sí. Como mencioné más arriba, la objeción de que la observación está cargada de teoría es en cierto modo irrelevante, pues lo que interesa es, en todo caso, si está cargada con la teoría correcta, la teoría adecuada o al menos con la mejor conjetura disponible. Cuando se señala que esta carga consiste en incluir supuestos antropocéntricos, se indica justamente que estamos proyectando una teoría incorrecta, una mirada antropomórfica sobre otras especies. Pero la mera mención de estos vicios argumentativos no resulta suficiente. En efecto, de un modo análogo, podemos acusar de antropocentrismo a quien se niega a interpretar ciertos gestos de un chimpancé como "frustración", pues -podemos afirmar- está proyectando una diferencia injustificada entre los animales humanos y los animales no humanos cercanos evolutivamente, es decir, está proyectando una concepción determinada y sustantiva del hombre, la cual anula por ejemplo toda atribución de "frustración" fuera del dominio humano.

Volviendo al cauce central de este trabajo, se trata de enfocar los problemas de atribuir a todo agente cognitivo las capacidades asociadas usualmente con el lenguaje. La objeción general, en este punto, es que no podemos hablar de la posesión de estas capacidades en ausencia del lenguaje, pues éste es una condición de su ejercicio. MacIntyre reconstruye del siguiente modo este proceder argumentativo:

Se toma como objeto de investigación una determinada capacidad humana: la capacidad para albergar pensamientos, creencias o para actuar movidos por razones, o la facultad para formular o utilizar conceptos. Entonces, se demuestra que, en contra de la opinión de algún filósofo precedente, el ejercicio humano de esa capacidad concreta requiere la 
posesión y el uso del lenguaje. Finalmente se concluye que, debido a que los animales no humanos no poseen lenguaje, o al menos el tipo de lenguaje requerido, carecen por ende de la capacidad o facultad en cuestión. ${ }^{5}$

Completando esta manera de argumentar, se sostiene que lo que existe es a lo sumo reacciones de estímulo y respuesta complejos, procesos descriptibles al modo behaviorista, procesos físico-químicos, procesos biológicos de complejidad diversa, etc., pero de ningún modo creencias, conceptos, inferencias auténticas. En esta línea, Brandom sostiene:

Responder sólo de una forma diferenciada y de modo consistente a las cosas que son rojas no es todavía ser consciente de ellas como rojas. La discriminación basada en la producción de respuestas reiteradas (como puede hacer una máquina o una paloma) categoriza los estímulos provocadores $y$, en ese sentido, los clasifica. Pero aún no es una clasificación conceptual...6

Esto último, es decir lo auténticamente conceptual, involucra para Brandom una habilidad inferencial expresada cabalmente en la práctica de dar y ofrecer razones, que requiere necesariamente del lenguaje.

Ahora bien, MacIntyre advierte con razón que el procedimiento general reconstruido incluye como parte central la demostración puntual de que determinada capacidad requiere del lenguaje. Respecto de cada capacidad se abre pues una discusión particular, a menudo sumamente compleja, acerca de su dependencia o independencia del lenguaje. Mi intención en este apartado no es abordar alguna de estas discusiones particulares, necesarias en cada caso para defender las alternativas teóricas adecuadas, sino mostrar lo que considero un supuesto implícito en quienes niegan la posesión de estas capacidades a seres no lingüísticos.

Me referiré a las variantes "débil" y "fuerte" de lo que consideraré el "argumento idealista" en especial relación con el lenguaje. En términos generales, este argumento se caracteriza por atribuir a todo agente cognitivo

${ }^{5}$ Ibid., pp. 26-27.

${ }^{6}$ Brandom, Robert, La articulación de las razones, Siglo XXI, Madrid, 2002, p. 21. 
las condiciones del conocimiento que se asume como válidas desde la perspectiva de quien realiza la atribución. En cada caso, consiste en atribuir al agente que es tema de la discusión filosófica alguna o algunas condiciones, capacidades, etc., que hacen posible la reflexión sobre dicho agente. Respecto del lenguaje, se trata de postular la validez general, para todo agente de conocimiento, de un aspecto central de la perspectiva del conocimiento humano complejo (y de la filosofía), precisamente la capacidad y ejercicio de un lenguaje natural e histórico.

La variante "débil" de este argumento tiene la siguiente forma (que subyace al argumento reconstruido por MacIntyre): de manera implícita, se absolutiza primero al lenguaje como condición para el conocimiento, el uso de conceptos, la posesión de creencias, etc., se advierte luego su ausencia en determinado agente, por ejemplo los animales no humanos, y se concluye de allí que este tipo de agente no posee en verdad la mencionada capacidad. Se afirma entonces que, respecto de estos agentes, no podemos hablar justificadamente de "tener creencias acerca de los otros", "realizar inferencias", “identificar objetos y personas", "comprender intenciones", etc. Como corolario, lo que nosotros, desde la perspectiva del lenguaje, describimos con esas expresiones, debería ser re-descrito en términos conductistas, fisicalistas, o en todo caso mediante un vocabulario adecuado a un reino no lingüístico, no humano o no racional.

Ciertamente este argumento no tiene la pretensión de ser idealista, pues la premisa inicial suele estar implícita, lo cual permite que se lo presente como el resultado de discusiones puntuales acerca de la relación entre el lenguaje y alguna de las mencionadas capacidades. Sin embargo, pienso que es una premisa idealista implícita lo que guía las conclusiones acerca del carácter no auténtico de diversas capacidades no lingüísticas.

Dicho con mayor precisión, es un argumento idealista débil por dos razones: primero porque corre el riesgo de resultar circular, asumiendo sin discutir una unión entre el lenguaje y alguna capacidad cognitiva particular. Así, se asume tanto una definición de lo que deberíamos considerar las capacidades ligadas con el lenguaje (qué es por ejemplo lo conceptual, o qué es tener una creencia) como la tesis correspondiente - y a menudo implicada en esta última - de que deberíamos considerarlas auténticas sólo si se las considera en su integración inseparable con el lenguaje. 
Segundo, el carácter débil de este argumento también consiste en que depende, a mi modo de ver, de una perspectiva idealista más fuerte. Sin este apoyo de fondo, a menudo implícito, la versión débil del argumento que estoy exponiendo debe admitir, tarde o temprano, que los agentes no lingüísticos ejercen de hecho alguna de las capacidades cognitivas descritas, aunque más no sea porque resultaría un trabajo arduo demostrar que cada una de ellas depende del lenguaje, o sería un trabajo sospechoso mostrar que cada una de estas capacidades carece de autenticidad (que no se trata de creencias en un sentido genuino, etc.). En efecto, el trabajo filosófico tiene la carga de mostrar que para capacidades tan diversas como tener creencias, realizar inferencias, comprender intenciones, etc., etc., precisamos del lenguaje. Bastaría con mostrar que una sola de estas capacidades puede ejercerse de manera genuina independientemente del lenguaje para mover la estantería del giro lingüístico en sus versiones más fuertes. Por esto, implícitamente, éste se apoya (necesita apoyarse) en un argumento de carácter más concluyente.

Se trata de la versión fuerte del argumento idealista respecto del lenguaje, que se puede formular del siguiente modo: la atribución de cualquiera de las mencionadas capacidades a agentes no lingüísticos es incorrecta porque se la realiza necesariamente mediante el lenguaje, en el medio del lenguaje, con el lenguaje como "esquema conceptual", o como quiera designarse al hecho de asumir la perspectiva del lenguaje como irrebasable. Según esto, entender el lenguaje como condición del resto de las capacidades cognitivas de todo agente cognitivo sería un resultado (débil) de entender que nuestra perspectiva en tanto científicos, filósofos o adultos parlantes, que es lingüística, se proyecta y abarca necesariamente (aunque de un modo que debe especificarse) toda la realidad, imponiendo al menos algunas de sus condiciones.

Así, al referirme a estas capacidades, al describir por ejemplo la atribución de capacidades cognitivas por parte de etólogos o científicos cognitivos a simios o niños pre-lingüísticos, estoy usando el lenguaje. De este modo, estoy determinando categorialmente, desde una meta-posición irrebasable, la propia descripción de la atribución y finalmente la atribución misma (las cualidades del agente que es objeto de la atribución). Se trata de una proyección categorial que abarca además el objeto conocido. 
De este modo, el idealismo de esta posición acerca del conocimiento determina una posición ontológica acerca de la relación entre el lenguaje y el mundo, que Gadamer expresa de esta manera:

La experiencia lingüística del mundo es "absoluta" (...) abarca todo ser en sí (...) La lingüisticidad de nuestra experiencia del mundo precede a todo cuanto puede ser reconocido e interpretado como ente. La relación fundamental de lenguaje y mundo no significa por lo tanto que el mundo se baga objeto del lenguaje. Lo que es objeto del conocimiento y de sus enunciados se encuentra por el contrario abarcado siempre por el horizonte del mundo del lenguaje. ${ }^{7}$

¿Es sostenible este argumento, ilustrado en su versión ontológica por la cita de Gadamer? Creo que no, por las siguientes razones. Es posible reconstruir las condiciones que rigen el conocimiento desde la perspectiva del lenguaje, pero es un error atribuir todas estas condiciones a todos los agentes cognitivos y finalmente a las cosas mismas, como es presuntamente el caso de Gadamer. Ante todo, porque algunas de esas condiciones son necesarias solamente para mi pensar actual, como mi propio cuerpo, mi lengua materna, etc. De este modo, algunas resultan contingentes para otros (alguien de otra cultura podría aprender el castellano que estoy usando y pensar también en esa lengua) o directamente no pueden concebirse como condiciones para otros ( $\mathrm{mi}$ propio cuerpo). Pero también pueden reconstruirse condiciones necesarias del tipo: creer algo implica no creer en su contrario. Esto significa que debo distinguir entre condiciones necesarias y no necesarias. Así como hay condiciones que sólo rigen para mí, puedo pensar que otros seres capaces de conocer no están sometidos a toda la serie de condiciones que rigen mi pensar actual. ${ }^{8}$ Los ejemplos de animales no

7 Gadamer, H.-G., Verdad y método, Sígueme, Salamanca, 1999, p. 539, el subrayado pertenece al autor.

${ }^{8}$ Desarrollé esta crítica en el IV Coloquio Latinoamericano sobre "Ética del Discurso. Interrogantes, articulaciones, convergencias", organizado por la Fundación ICAI_A (Río Cuarto) en noviembre de 2009 ("Conocimiento, reflexión y lenguaje en la transformación pragmático-trascendental de la filosofía kantiana". En: Michelini /Salerno/Crelier (Comps.), Ética del Discurso. Aportes a la ética, la política y la semiótica. Río Cuarto: Ediciones del ICALA/Fundación Alexander von Humboldt, 2010.) Los comentarios y críticas de Alberto Damiani, Daniel Kalpokas y Adrián Bertorello, 
humanos y niños pequeños sugieren justamente que para ellos es posible conocer sin lenguaje.

Con otras palabras, las condiciones necesarias para ejercer la ciencia, la filosofía o hablar un lenguaje no son todas ellas condiciones necesarias de toda acción y capacidad racional, conceptual o perceptiva. Bertrand Russell expresa los riesgos de la perspectiva que estoy criticando del siguiente modo: "a menos que seas bastante auto-consciente acerca de los símbolos, a menos que estés lo suficientemente prevenido acerca de las relaciones del símbolo con aquello que simboliza, te encontrarás atribuyendo a la cosa propiedades que sólo pertenecen al símbolo"9.

Creo que ése es justamente el error idealista que describo. El resultado que aquí interesa es la atribución de todas las condiciones del conocimiento lingüísticamente mediado (el símbolo, en términos russellianos) a toda clase de actividad cognitiva (la cosa). Ahora bien, es importante resaltar que se trata de un error cuando la atribución abarca todas las condiciones, pero que puede resultar un argumento válido respecto alguna de ellas. En nuestro caso, se trata de condiciones lingüísticas, por lo que la crítica al argumento idealista puede tomar formas como la siguiente: "Si bien las oraciones que usamos para atribuir pensamientos tienen componentes, nuestras atribuciones no presuponen una atribución a priori de estos componentes"10.

El argumento idealista sirve como apoyo, entonces, para una versión lingüística (las condiciones del conocimiento en general incluyen el lenguaje). La crítica a la versión fuerte del argumento idealista le resta apoyo a la versión débil. Si no hay razones para atribuir al objeto de análisis -los agentes capaces de conocer- todas las condiciones necesarias para realizar esta atribución -condiciones que involucran el lenguaje-, entonces no hay razones para considerar que el lenguaje interviene necesariamente en todo

dieron lugar al artículo "Reflexión y lenguaje. Perspectivas de la transformación pragmático-trascendental de la filosofía kantiana", en la Revista de Filosofía y Teoría Política, Universidad Nacional de La Plata, volumen 43, 2012.

9 Russell, Bertrand, The Philosophy of Logical Atomism, Routledge, London / New York, 2010, p. 10. La traducción me pertenece.

10 Glock, Hans, "Can Animals Judge?", Dialectica, Vol. 64, N 1, 2010, p. 20. La traducción me pertenece. 
proceso cognitivo. En suma, es posible conservar cierto escepticismo acerca de que las condiciones lingüísticas que permiten una reflexión científica o filosófica deben atribuirse también a todo agente que conoce y actúa.

Esto no quita que sea lícito atribuir algunas de estas condiciones, y, en términos más generales, se pone en evidencia la necesidad de explorar en general -más allá de las discusiones puntuales entre diversas capacidades y el lenguaje- la relación entre lo pre-lingüístico y lo lingüístico.

\section{La relación entre lo pre-lingüístico y lo lingüístico y el problema de la "incongruencia"}

El idealismo de fondo que he intentado sacar a la luz resulta ciertamente cuestionable. Sin embargo, es posible defender una versión del mismo que tome en cuenta esas críticas. Se trata de sostener que, aunque no todas las condiciones de quien conoce mediante el lenguaje pueden atribuirse legítimamente a todo agente de conocimiento, todo proceso cognitivo y todo conocimiento es virtualmente expresable mediante el lenguaje. Tanto lo comprendido y/o conocido por seres lingüísticos como nosotros como el modo en que nos representemos a otros agentes de conocimiento y sus procesos cognitivos, tiene que ser virtualmente expresable mediante el lenguaje. Esto es así incluso cuando el agente que es objeto de nuestras atribuciones teóricas no sea un agente lingüístico.

El argumento para sostener esto es, como se mencionó, idealista y parte de asumir plenamente la perspectiva del lenguaje. Esta perspectiva determina que no podemos afirmar que existe un fenómeno de conocimiento (algo conocido o alguien que conoce) si no podemos formular el contenido de este fenómeno mediante palabras. Por supuesto, es posible que dos agentes carentes de la capacidad lingüística se comprendan entre sí en base a que comparten una forma de vida, sin que nosotros accedamos al sentido de lo comprendido. Pero en tanto seamos capaces de detectar el fenómeno aludido, de ver en él un proceso de comprensión -en este caso de comprensión mutua- y de detectar allí el funcionamiento de capacidades cognitivas, seremos capaces de "lingüistificarlo" (al modo de "el delfín cree que $\left.p^{\prime}\right)$. La tesis puede especificarse señalando que lo comprendido es, 
desde nuestra perspectiva lingüística, virtualmente expresable mediante el lenguaje $\mathrm{e}^{11}$.

La idea de que el lenguaje, dicho en términos que remedan la autoconciencia kantiana, tiene que poder actualizar todo fenómeno de conocimiento, deja abierta la cuestión del modo concreto en que esto se realiza. Si lo actual -el lenguaje- es capaz de actualizar lo virtual prelingüístico -el conocimiento no mediado aún por el lenguaje- debe existir entonces una relación posible entre las dos dimensiones (pues se trata de dos ámbitos que, en su rendimiento actual, son diferentes). ¿En qué consiste concretamente la relación entre el sentido pre-lingǘstico y su correspondiente lingüístico? ¿Cómo es posible que algo común tenga dos naturalezas diferentes? ¿Cómo se constituye el lenguaje a partir de capacidades y sentidos o significados previos?

Estas preguntas abren discusiones que exceden el marco de este trabajo. Sin embargo, intentaré esbozar una posible vía para iniciar su tratamiento. Es importante señalar que cuando se habla de lo virtual prelingüístico se tiene en mente su posible relación con el lenguaje, pero las capacidades cognitivas y la comprensión efectiva ya están funcionando plenamente aun sin lenguaje. De este modo, el lenguaje se enfrenta con una dimensión que, a su modo, tiene un carácter actual, a una positividad fenoménica independiente.

11 En el Heidegger de Ser y Tiempo se encuentra esta concepción de que existe un ámbito de significatividad práctica que es de naturaleza ante-predicativa y que puede articularse mediante enunciados. Si bien el lenguaje en sentido amplio, entendido como la significatividad en general, es irrebasable para todo ser-en-el-mundo, el lenguaje en sentido estrecho, entendido como una lengua convencional y articulada que permite formulaciones de naturaleza proposicional, es algo derivado y, en tal medida, no necesario. Esto se advierte por ejemplo en la siguiente cita: "El trato circunspectivo-interpretante con el ente a la mano del mundo circundante, que lo 've' en cuanto mesa, puerta, coche o puente, no tiene necesidad de exponer también en un enunciado determinativo lo circunspectivamente interpretado. Toda simple visión antepredicativa de lo a la mano ya es en sí misma comprensorainterpretante." Cf. Heidegger, Martin, Ser y tiempo. Traducción, prólogo y notas de Jorge Eduardo Rivera C., Santiago de Chile, Editorial Universitaria, 1998 (1927), p. 173). 
Con otras palabras, el lenguaje expresa algo previo, la materia prelingüística (creencias, inferencias, etc.). Desde el punto de vista del lenguaje, lo expresado está implícito, aunque desde el punto de vista de lo expresado se encuentre ya explicitado a su modo, en conductas, hábitos o procesos cognitivos. La actualización de un contenido virtual se entiende, pues, como un proceso de explicitación. El sentido de una acción extra-lingüística es explicitado mediante palabras porque ya posee un significado, tanto para quien actúa como para un observador.

Esto conduce al siguiente problema. Para cada fenómeno prelingüístico existiría una contrapartida lingüística posible, un modo lingüístico de empezar al menos la "lingüistificación" de este fenómeno. ${ }^{12}$ Pero el contenido pre-lingüístico no es, como hemos asumido, exactamente de la misma naturaleza que su contraparte lingüística (ante todo, no es ya lingüístico). Como consecuencia, resulta problemático usar el lenguaje exactamente del mismo modo para atribuir contenido lingüístico a un agente lingüístico (Juan cree que $p$ ) que para atribuir contenido no lingüístico a un agente pre-lingüístico (el delfín cree que $p$ ), actualizando así un contenido virtual. Así lo formula Glock en referencia a la atribución de creencias:

La distinción entre distintas clases de contenidos parece ir contra atribuir una y la misma creencia a animales y a humanos. Sugiere que "Tanto Sara como el perto creen que p" no es tanto una falsedad como un zeugma. Porque "Sara cree que p" resulta en "Sara está en una relación de creencia con el pensamiento que p", mientras que "El perro cree que p", resulta en "El perro está en una relación de creencia con el proto-pensamiento que $\mathrm{p} " 13$.

Puede afirmarse que este problema se repite con cada atribución de capacidad cognitiva. Así, si admitimos que lo conceptual puede también entenderse como una habilidad no lingüística de naturaleza cognitiva, que

12 Esto, sin embargo, tiene sus límites, en tanto no todo saber práctico parece formulable mediante el lenguaje. Sé cómo levantar mi brazo, pero este saber no es traducible en palabras. No abordaré el problema que representan estos límites pues me interesa resaltar ahora la capacidad positiva del lenguaje para expresar lo prelingüístico.

${ }^{13}$ Glock, H., Op. cit., p. 16. 
permite por ejemplo la identificación de sustancias, atribuir capacidad conceptual a animales mediante formulaciones proposicionales resulta incongruente, y el mismo caso se presenta con capacidades específicas como la de realizar inferencias, comprender las intenciones, etc. (X infiere que $\mathrm{p}$, $\mathrm{X}$ comprende la intención que $\mathrm{p}$, etc.). Siempre que formulamos proposicionalmente la actitud cognitiva de un agente no lingüístico estamos al parecer falseando los fenómenos, pues expresamos algo que necesariamente no puede ser así. En efecto, los agentes no lingüísticos no pueden mantener ninguna relación con proposiciones ${ }^{14}$.

La relación de un animal no humano o un niño pre-lingüístico con lo creído, comprendido, inferido, sería una relación con algo diferente a la relación que, como seres lingüísticos, tenemos con el contenido de nuestras creencias, inferencias, etc. Sin embargo, si es correcto pensar, a partir de la observación empírica, que existe conocimiento en estos agentes, debe pensarse que existe también en algún aspecto una identidad entre ambas relaciones.

El concepto de constitución puede sugerir un modo de abordar este problema. Se trata considerar que lo pre-lingüístico es una materia que lo lingüístico configura. Dado que se trata de la misma materia, se conserva la identidad, pero la configuración proporciona algo nuevo. Así, existe por un lado identidad entre materia y forma, pues lo creído, inferido, comprendido, etc., es lo mismo antes y después de su actualización mediante el lenguaje. Pero existe también una no-identidad, pues el lenguaje, al expresar lo prelingüístico, opera como una forma que ofrece nuevas posibilidades cognitivas.

La expresión de algo previo, y la constitución del lenguaje en base a materia previa de sentido, ponen de manifiesto así que el lenguaje aporta algo nuevo. El novum, a mi modo de ver, incluye entre otras funciones que me parece relevante destacar la capacidad de expresar y explicitar razones. Esta capacidad argumentativa, que me interesa por su relevancia para la ética

${ }_{14} \mathrm{Al}$ menos si pensamos que éstas se vehiculizan necesariamente mediante una lengua natural. En todo caso, si se admite que las proposiciones pueden vehiculizarse en el caso animal en otro tipo de soportes, sigue en pie el problema de la congruencia, pues se trataría de una relación con algo diferente al lenguaje natural humano. 
discursiva, consiste en una dimensión propiamente lingüística irreductible a las esferas cognitivas pre-lingüísticas que estamos admitiendo. Así, el contenido de una creencia por parte de un delfín no puede desarrollarse sin lenguaje en la justificación de esa creencia (es una cuestión empírica si el animal en cuestión ha adquirido algún lenguaje que lo permita).

$\mathrm{Si}$ bien las capacidades pre-lingüísticas son normativas, pues los agentes esperan que sus creencias, inferencias, etc. sean correctas (esto se evidencia en su conducta), lo que no pueden hacer es justificar o criticar su contenido explícitamente, es decir, no meramente como algo inferible de su conducta. El medium del lenguaje convencional, adquirido mediante socialización en una comunidad lingüística, se revela pues como una potente herramienta que permite explicitar el contenido del conocimiento prelingüístico, resignificarlo, ponerlo en relaciones que podemos considerar "holistas" y, finalmente, fundamentarlo y criticarlo. Resultaría absurdo requerir esto de un agente a-lingüístico no porque le falte desarrollar alguna de sus capacidades cognitivas, sino porque le falta algo nuevo y diferente. No existe ninguna capacidad pre-lingüística que pueda desarrollarse de modo que resulte en un lenguaje, aunque el conjunto de estas capacidades es lo que conforma la infraestructura del mismo.

\section{Observaciones finales sobre la aplicación de la ética discursiva a agentes pre-lingüísticos}

Volviendo a las intenciones centrales de este trabajo, referidas a la ética discursiva, es posible ver ahora que la relación entre lo pre-lingüístico y lo lingüístico permite pensar que los presupuestos éticos también podrían, en algunos casos, aplicarse a agentes pre-lingüísticos. En efecto, podemos pensar que el lenguaje es capaz de actualizar capacidades cognitivas complejas y "superiores", ante todo explicitando las razones implícitas en la conducta y el conocimiento pre-lingüístico.

Se refuerza la ética discursiva porque se justifica el carácter expansivo del principio del discurso. Si bien la ética discursiva enuncia condiciones suficientes para ser un agente moral, no se trata de condiciones necesarias, pues es posible encontrar otros fundamentos para atribuir carácter moral a un agente, como la presencia de determinadas facultades de conocimiento. 
En suma, reforzar la relación entre lo virtual y lo actual, y explicarla, justifica ampliar el universo moral con argumentos atendibles, como resumidamente se ve en los siguientes puntos:

1. La acción no lingüística realizada por un agente con capacidad lingüística queda plenamente dentro de la esfera de la moralidad, pues sus actos tienen que poder justificarse del mismo modo que los actos de habla.

2. Es posible fundamentar un gradualismo moral que otorgue un punto mayor de apoyo a la intuición según la cual la esfera de la moralidad incluye a los niños pre-lingüísticos y a quienes han perdido la capacidad de hablar.

3. Se hace posible ampliar este gradualismo, justificando con esto las intuiciones morales que consideran la presencia de derechos morales en al menos algunos animales cercanos biológicamente al ser humano.

Recibido: 8/2012. Aprobado: 10/2012. 